\title{
International Congress on Theoretical Physics at Kharkov
}

A

International Congress on Theoretical Physics was held at Kharkov on May 19-23 under the auspices of the Ukraine Physico-Technical Institute, and the chairmanship of L. Landau. At the official opening of the Congress, Prof. Niels Bohr, of Copenhagen, delivered a lecture on "Causality in Physics". Papers were read by E. J. Williams, E. Lifschitz, M. S. Plesset, V. A. Fock, L. Landau, I. Waller, J. Frenkel, M. Bronstein, J. Solomon, I. Tamm and L. Rosenfeld; and Niels Bohr joined in the discussions.

Williams described the general state of research on the scattering of hard $\gamma$-rays, and his recent experiments with thin scatterers. If sufficiently thin scatterers are used, the range of the electrons produced by the $\gamma$-rays is sufficient to enable them to escape from the foil and therefore to avoid annihila. tion except far away from the foil. In this way the annihilation radiation is separated from the other part of the radiation. It is found by this method that the annihilation radiation definitely exists and constitutes the whole of the scattered radiation of $0.5 \times 10^{8}$ volts. From observations on the variation of the scattered intensity with the thickness of scatterer, an approximate estimate of the energy of the positive electrons may be made. The fraction of low energy positive electrons proves to be unexpectedly high, and may be explained by the production of double pairs. In the discussion, Frenkel suggested that the low energy of some positive electrons might be due to the production of pairs by a Compton effect. Gray and Tarrant's result that the energy of scattered radiation is practically equal to that of the absorbed incident radiation could be explained in the same way. Williams described a method of obtaining Heitler and Sauter's formula for the energy lost by an electron in radiative collisions with a nucleus. The perturbing field of the nucleus is analysed into harmonic components and the KleinNishina formula is applied to the scattering of the separate components.

In another paper, Williams described some evidence for the existence of the negatron, provided by observations of Kunze on cosmic rays. Kunze's Wilson chamber photographs seem to show that the high energy particles of cosmic radiation produce about 20 ions per $\mathrm{cm}$. in normal air. If the particles were electrons, about thirty-five would be expected; if they were protons, twenty to twentyfive. As some of Kunze's tracks have a negative curvature, they may be due to negatrons.

Plesset discussed Dirac's theory of the positive electron and the developments made by Fock and by Carlson and Oppenheimer. The difficulties in these formulations connected with invariance was pointed out. Dirac's recent procedure is the only one so far which possesses the required invariant properties, but it is only approximate. The significance of the approximation was explained.

In another paper, Plesset described an application of quantum electrodynamies to the determination of the proper energy of a vacuum with the theory of filled negative energy states. Transitions are considered from the initial state of the vacuum distribution to intermediate states in which an electronpositron pair and a quantum of radiation are present; then transitions are considered from these inter- mediate states back to the initial state. The intermediate states may be given the picturesque interpretation of representing fluctuations in the vacuum distribution. If the summation over all intermediate states is performed, an expression is secured which represents the coupling of the initial state with itself by means of an effective self-interaction energy. This proper energy of the vacuum may be readily found to be infinite. An analogous calculation may be made for the case of the presence of an external field.

Lifschitz discussed the production of electronic pairs by a collision of two particles. The crosssection is calculated when the velocities are near to the velocity of light. For this case the particles may be assumed as moving rectilinearly without interaction and the effect is then due merely to the superposition of both fields. The calculation shows that the whole cross-section increases with the cube of the logarithm of the energy of the colliding particles.

Waller discussed the recoil of rays scattered by free electrons. It is well known that the theories based on point electrons do not make possible an adequate treatment of this problem. The appearance of infinities in the problem of the recoil of rays from bound electrons has been avoided by special devices (Dirac, Landau, Weisskopf, Wigner and others). In this way, analogies with classical theory have been obtained. The appearance of infinities in the problem of scattering by free electrons may be avoided in the calculation of the recoil (to the first approxima. tion) by a simple device based on Dirac's theory of rays.

Fock described his mathematical developments of Dirac's theory of the positive electron. This work was published in Russia in 1933 and has not yet become sufficiently known. He explained that it simplifies the mathematics, but does not alter any of the physical difficulties in the theory; it has only made them more accessible.

Tamm discussed the deduction of exchange forces between neutrons and protons from Fermi's theory of $\beta$-radioactivity based on the assumption that transmutations of a neutron into a proton and vice versa are possible, and are accompanied by the birth or disappearance of an electron and a neutrino. Consider two heavy particles, $a$ and $b, a$ being in a neutron and $b$ in a proton state. If $a$ becomes a protor and $b$ a neutron, the energy remains unchanged. Now those two degenerate states of the system may be linked by a two-step process-the emission of an electron and a neutrino by the neutron (a), becoming a proton, and the ensuing reabsorption of these light particles by the proton $(b)$, becoming a neutron. The energy of the system is in general not concerned in the intermediate state. The emission and reabsorption of a positron and neutrino may also take place. In this way the two degenerate states of the system considered are split into two energy states differing by the sign of the exchange energy. Calculation shows that if the difference of masses of the neutron and of the proton is larger than the sum of the masses of an electron and a neutrino, the emission of light particles by a heavy particle may take place without a violation of the conservation of energy. But the corresponding value of the exchange energy may be shown to be far too 
small. The negative result indicates that either the Fermi theory needs a substantial modification or that the origin of the forces between neutrons and protons does not lie, as would correspond to the original suggestion of Heisenberg, in their transmutations considered in detail by Fermi.

Rosenfeld described researches made in collaboration with Cambresier on dissociative equilibrium in stellar atmospheres. The number of molecules of a given kind in the atmosphere of a star can be calculated as a function of the effective temperature and surface gravity of the star on the assumption of dissociative equilibrium. In this computation it is essential to take into account the variation of pressure in the different layers of the atmosphere. The pressure at the base of the atmosphere may be calculated from the general absorption, by a method first used by Milne and Chandrasekhar. The treatment of concrete cases necessitates assumptions on the relative abundances of the atoms taking part in the reactions, but the results are quite insensitive to such assumptions. The equilibria of $\mathrm{TiO}, \mathrm{ZrO}$, and of the carbon combinations $\mathrm{CN}, \mathrm{CH}, \mathrm{CO}, \mathrm{C}_{2}$, have been computed in two different cases: when $O$ is much more abundant than $\mathrm{C}$, and vice versa. It is seen that the first case corresponds to the main sequence, the second to the branch of carbon stars, and that simply on this assumption a satisfactory agreement is obtained with the observed variations of intensity of the corresponding bands with spectral type and surface gravity (giant or dwarf character of the stars).

The members of the Congress had the opportunity of visiting many interesting institutions in Kharkov, Moscow and Leningrad. The laboratories of the Ukraine Physico-Technical Institute, and the Physical Institutes in Leningrad and Moscow were attractive, on account of the youthful enthusiasm of the staffs, besides the variety of the researches in progress. The Dzerzhinsky School for Orphans at Kharkov, organised and supported by personal subscriptions from the members of the G.P.U., contains four hundred boys and girls. They live with a large degree of self-government, and are trained in three magnificent factory-workshops, one of which is for the complete manufacture of cameras on the Leica model, including the lenses. A kolkhoz of three thousand acres supporting seven hundred persons was also very instructive. At the Kharkov Tractor Works, tractors of the McCormick type could be seen running off the assembling conveyor at the rate of 140 per day. In Moscow the constructive works of the underground railway were prominent. The members of the Congress will remember for a long time the interest of their visit and the hospitality of their hosts.

\section{Aberdeen Meeting of the British Association}

$\mathrm{I}^{\mathrm{N}}$ a previous article (Nature, 133, 673, May 5), reference was made to the suitability of Aber. deen as a centre for excursions, and advantage has been taken of the city's position in this respect by the local committee for the Aberdeen meeting of the British Association to be held on September 5-12. Arrangements for excursions to places of historical interest through some of the most striking natural scenery in Scotland have now been completed. The Committee did not dare to hope that it could call upon the members of the Association to repeat the experience of its predecessors of nearly eighty years ago, where some of the excursions occupied the better part of two days. It has therefore arranged that the excursions taking place on the Saturday of the meeting will start at a comfortable time after breakfast and arrive back before dinner. The Committee has been fortunate in securing the services of authorities on the different areas and places of interest to be visited to write descriptive articles on the general excursions, and these articles will add to the enjoyment of these excursions. Arrangements have also been made for guides where necessary, to accompany the members and to give information concerning the different places visited.

Probably the most interesting excursion for those who enjoy natural scenery is the Highland excursion. This starts at 9.20 a.m. by train through the cultivated parts of Aberdeenshire and Banffshire, thence into the valley of the Spey, which is followed, first westwards to Boat of Garten, then southwards to Aviemore where, looking eastward, there is a striking view of the Cairngorm range of mountains. Visitors should notice a V-shaped depression between Ben MacDhui and Braeriach, which marks the position of the highest mountain pass in Scotland-Lari Gghru. After leaving Aviemore, the train proceeds towards Inverness, passing Carr Bridge and Tomatin to
Culloden Moor, where the members will leave the train and proceed by bus to the battlefield. There, local guides will join the party and give a short account of the battle and the objects of interest, including the famous Clava Cairns, on the Moor. The visitors will then proceed to Inverness, alighting on the Castle Hill. In Inverness they will be entertained by the Provost, Magistrates and Town Council to tea. After an interval, which can be utilised for seeing some of the places of interest in the city, the train will return from Inverness by the coast, from which there is a striking view of the mountains of Ross-shire, Sutherland and Caithness, the most prominent among these being probably the cone of Morven, which can be seen for a long way along the coast.

From a historical point of view, an interesting excursion is that which has been arranged to leave Aberdeen at 9.40 a.m. by train to Elgin, arriving there at noon. There, the members of the Association will be welcomed by the Lord Provost, Magistrates and Town Council of Elgin, and will be entertained to lunch. After lunch they will proceed by charabane through the ancient province of Moray, which abounds in historical remains. The itinerary will be from Elgin Cathedral to Spynie Castle, Lossiemouth, Duffus Castle, Kinloss Abbey and Pluscarden Abbey. There will be an interval at Pluscarden Abbey, where tea will be provided near the ruins. Thereafter the party will return to Elgin, whence they will entrain for Aberdeen at 5.15 p.m., returning by a different route.

An excursion has also been arranged by motor. bus leaving Marischal College at 10 a.m., and proceeding along the North Deeside Road through the Pass of Ballater and Braemar to the Linn of Dee. On the return journey from the Linn of Dee, a halt will be made at Braemar for lunch. The party will 